\title{
Squeeze-film damping characteristics of cantilever microresonators for higher modes of flexural vibration
}

\author{
S. Chaterjee, G. Pohit*
}

Department of Mechanical Engineering, Jadavpur University, Kolkata 700032, INDIA

"Corresponding Author: e-mail: gpohit @ vsnl.net

\begin{abstract}
Squeeze-film characteristics of electrostatically actuated microcantilevers observed under large DC load coupled with small AC component are presented for the first three flexural modes of vibration of the resonator operating in different ambient pressure conditions. A semi-analytical model of an electrostatically actuated microcantilever beam is developed taking into account the dependency of the effective viscosity on variable gap spacing through the variable Knudsen number. The quality factors of the system are also obtained numerically using the coupled field FE analysis software ANSYS. A comparison of the results up to the pull-in instability shows excellent agreement for damping characteristics obtained by both the semi-analytical and FE methods. The effects of large DC bias voltages on quality factor and resonance frequency are found to differ considerably for the different flexural modes of vibration of the resonator. Under rarefied flow conditions, variations in ambient pressure significantly influence the squeeze film damping characteristics for the entire range of DC polarization. A comparison between compressible and incompressible flow models predicts notable difference in the squeeze-film characteristics.
\end{abstract}

Keywords: MEMS, Electrostatic actuation, Squeeze-film damping, Rarefied flow, Resonant sensor.

\section{Introduction}

Resonantly actuated microstructures, referred to as micro-resonators, are an integral part of many MEMS devices such as RF filters [Nguyen 2004], chemical and mass sensors [Thundat et al. 1995], physical sensors [Oden et al. 1996], and atomic force microscopes [Maali et al. 2005]. In case of an electrically actuated resonant sensor, electric load composed of a DC bias voltage and an AC component excites the structure. In case of small DC bias voltage the static bias deflection may be neglected but should be accounted for voltage large enough to cause non-uniform gap spacing between the cantilever electrode and the ground plane [Batra et al. 2008]. Moreover, large DC voltages cause softening of the system [Nathanson et al. 1967]. In practice, the electromechanical system is complicated by the effects influenced by the gas surrounding the system. The gas damping may be of the same order of magnitude as the electric and mechanical forces, and thus it needs to be accounted for in the design of the devices. Viscosity, non-uniform gap spacing, and, the operating frequency influence the pressure distribution in the air-gap thus affecting the squeeze-film damping/stiffening effects. It is, therefore, essential to effectively model the electro-mechanical coupling of the system and the damping effects to correctly predict the most important design characteristics of the resonator, namely, the resonant frequency and the quality factor.

The damping of electrically actuated microstructures vibrating in presence of gas trapped in the narrow gap is dominated by squeeze-film damping [Hamrock 1994]. The gas in the gaps affects the system in two different ways. The gas may be compressed due to movement of the mechanical parts of the system and, thus, cause a spring force opposing the movement. The gas also flows sideways in the gap which in turn dissipates the movement. Further, the gas flow is rarefied for MEMS devices operating at low pressure with a narrow gap between the movable electrode and the ground plane. Modeling of such a system is quite involved since the dynamic problem is coupled in the electrostatic, structural, and fluid domains. Several approaches [Langlois 1962; Blech 1983; Darling et al. 1998; Veijola et al. 1995] assumed the microstructure to be rigid and analyzed only the structural and fluid domains. When the microstructure is assumed to be rigid, the Reynolds equation is decoupled and, on linearization, simpler 
expressions for the damping coefficient can be obtained. Newell [1968] used cantilever resonators to study the effect of damping in different pressure regimes. In the regime of high pressure, viscous damping was found to be dominant. Dynamics of damped flexible structures actuated by transient electric loads have been reported in open literature [McCarthy et al. 2002; Krylov and Maimon 2004]. For flexible structures vibrating even in the first mode of vibration, the gap is non-uniform and the elasticity equation that gives the dynamic displacement under electrostatic load is coupled to the Reynolds equation. McCarthy et al. [2002] modeled a cantilever switch and assumed the pressure distribution in the gap to be separable being the product of a parabolic function along the beam width and an unknown function along the beam length which is determined during solution. Krylov and Maimon [2004] assumed the flow in the gap to be incompressible and studied the damped transient characteristics of a cantilever coupled to a plate at the free end. Pandey and Pratap [2007] obtained the damping characteristics for the first three flexural modes of vibration of the resonator. The linearized compressible Reynolds equation coupled with the elastic beam equation was solved, using Green's function approach. However, they neglected the static deflection due to the DC load. Subsequently, they solved the coupled structural-fluid problem using the modal projection technique available in ANSYS. Zhang et al. [2004] neglected the static bias deflection and modeled the resonator as a flexible double-clamped microbeam vibrating in the first mode of vibration. The pressure distribution in the gap was expressed in the form of a trigonometric series. Damping of electrically actuated microbeam under static bias deflection was analyzed by Li et al. [2007] but the model does not consider the dependency of the effective viscosity on the gap height which is no longer uniform for a statically deflected beam. Paci et al. [2006] experimentally investigated the damped characteristics of free-free beam resonators resonating on the first and third modes. High quality factor was obtained for the free-free third-mode resonator. The problem of large DC bias voltage actuation of microplates under different gas pressures was studied by Younis and Nayfeh [2007]. The static bias deflection for the microplate was obtained using the beam model and a perturbation method was used to obtain the pressure distribution in terms of the structural mode shapes. However, the perturbation approach does not apply to cantilever plates because of relatively small fundamental natural frequencies.

In this paper, the microresonator modeled as a cantilever beam actuated by large DC load and small AC component is analyzed using a semi-analytical method. The dynamic problem coupled in the electrostatic, structural, and fluid domains is formulated by coupling the Euler-beam equation, incorporating the electrostatic force terms and the squeeze-film damping terms, with the linearized compressible Reynolds equation. The static deflection under DC bias voltage is obtained using a reduced order model (ROM) based on the method of Galerkin discretization. The present model takes into account the dependency of the effective viscosity on variable gap spacing through the variable Knudsen number. The quality factors of the system are also obtained numerically using the coupled field FE analysis software ANSYS. A comparison of the results up to the pull-in instability shows excellent agreement for damping characteristics obtained by both the semi-analytical and FE methods. Design characteristics of the resonator operating under rarefied and inertia-less flow conditions are found to be significantly influenced by DC bias voltage for the analyzed modes of vibration.

\section{Governing Equations}

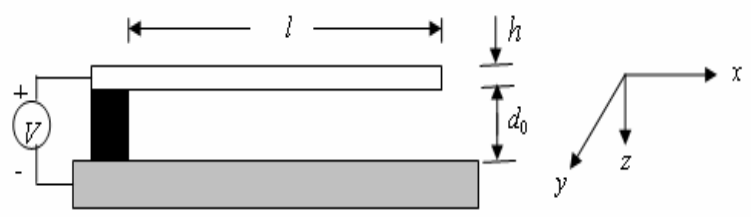

Figure 1. A schematic diagram of an electrostatically actuated microcantilever beam model.

The model (Fig. 1) of a MEMS resonant sensor shows a damped cantilever beam of length $l$, width $b$, thickness $h$ separated from the ground plane by a small initial air-gap of $d_{0}$. When subjected to a driving voltage $V(t)$ comprising of a DC bias voltage $V_{D C}$ and a small AC component $v(t)$ with $V_{D C}>v(t)$, the beam undergoes small amplitude motions around the static deflected position. Let $w(x, t)$ denote the transverse displacement of the beam being dependent on the position $x$ along the beam length and time $t$. Following the elastic beam theory, and, adding terms representing electrostatic excitation and squeeze-film effect, the governing equation of the one- dimensional Euler-beam for small air-gap to length ratio is given by

$$
\rho A \frac{\partial^{2} w}{\partial t^{2}}+E I \frac{\partial^{4} w}{\partial x^{4}}=\frac{1}{2} \frac{\varepsilon_{0} b V^{2}}{\left(d_{0}-w\right)^{2}}-f_{d}(x, t)
$$

with the boundary conditions as $w(0, t)=(\partial w / \partial x)_{x=0}=0$, and, $\left(\partial^{2} w / \partial x^{2}\right)_{x=l}=\left(\partial^{3} w / \partial x^{3}\right)_{x=l}=0$

where $\rho$ is the density of the beam material; permittivity constant for free space $\varepsilon_{0}=8.854 \times 10^{-12}, F^{-1}$; plate modulus for wide beams $E=E^{\prime} /\left(1-v^{2}\right), E^{\prime}$ is the Young's modulus, $v$ is the Poisson's ratio. For slender beams, $E=E^{\prime}$. The first term on 
the right hand side of Eq. (1a) represents the excitation force per unit length while the second term represents the force acting on the beam owing to the pressure of the squeezed gas film between the beam and the ground plane. The one-dimensional force due to squeeze-film damping is obtained by integrating the two-dimensional pressure distribution along the width of the beam, and, is given by

$$
f_{d}(x, t)=\int_{0}^{b}\left[p(x, y, t)-p_{a}\right] d y
$$

where $p(x, y, t)$ is the absolute pressure in the gap and $p_{a}$ is the ambient pressure.

\subsection{Compressible flow model}

For inertia-less (Reynolds number less than unity) viscous motion within small gaps and the motion of the beam being restricted to normal approach, the pressure $p(x, y, t)$ under isothermal conditions is governed by the nonlinear compressible Reynolds equation [Hamrock 1994]

$$
\frac{\partial}{\partial x}\left(\frac{1}{12 \mu_{\text {eff }}} g^{3} p \frac{\partial p}{\partial x}\right)+\frac{\partial}{\partial y}\left(\frac{1}{12 \mu_{\text {eff }}} g^{3} p \frac{\partial p}{\partial y}\right)=\left(g \frac{\partial p}{\partial t}+p \frac{\partial g}{\partial t}\right)
$$

with trivial boundary conditions as $p(x, 0, t)=p(x, b, t)=p(l, y, t)=p_{a}, \partial p(0, y, t) / \partial x=0$

where the non-uniform gap spacing $g(x, t)=d_{0}-w(x, t)$; variable effective viscosity $\mu_{\text {eff }}=\mu /\left(1+9.638 K n^{1.159}\right)$ is considered to account for the rarefied gas effects [Veijola et al. 1995]; the variable Knudsen number $K n=\lambda_{a} / g ; \lambda_{a}=\lambda_{0} p_{0} / p_{a}$ is the mean free path at ambient pressure $p_{a}$; the mean free path $\lambda_{0}$ at standard temperature and pressure $p_{0}=1.013 \times 10^{5} \mathrm{~Pa}$ conditions is about $65 \times 10^{-9} \mathrm{~m} ; \mu$ is the dynamic viscosity of air under standard temperature and pressure.

Using Eq. (2) and the nondimensional variables $\bar{x}=x / l, \bar{y}=y / b, \bar{w}=w / d_{0}, \bar{g}=g / d_{0}, \bar{p}=p / p_{a}, \bar{t}=t / s$, $s=l^{2} \sqrt{\rho A} / \sqrt{E I}$, Eq. (1) can be expressed as

$$
\begin{aligned}
& \frac{\partial^{2} \bar{w}}{\partial \bar{t}^{2}}+\frac{\partial^{4} \bar{w}}{\partial \bar{x}^{4}}=\frac{1}{2} \frac{\alpha V^{2}}{(1-\bar{w})^{2}}-\hat{P} \int(\bar{p}-1) d \bar{y} \\
& \bar{w}(0, \bar{t})=(\partial \bar{w} / \partial \bar{x})_{\bar{x}=0}=0, \text { and, }\left(\partial^{2} \bar{w} / \partial \bar{x}^{2}\right)_{\bar{x}=1}=\left(\partial^{3} \bar{w} / \partial \bar{x}^{3}\right)_{\bar{x}=1}=0
\end{aligned}
$$

and, Eq. (3) can be expressed as

$$
\begin{aligned}
& \frac{\partial}{\partial \bar{x}}\left[\frac{1}{\mu_{\text {eff }}}(1-\bar{w})^{3} \bar{p} \frac{\partial \bar{p}}{\partial \bar{x}}\right]+\beta^{2} \frac{\partial}{\partial \bar{y}}\left[\frac{1}{\mu_{\text {eff }}}(1-\bar{w})^{3} \bar{p} \frac{\partial \bar{p}}{\partial \bar{y}}\right]=\frac{12 l^{2}}{d_{0}{ }^{2} p_{a} s}\left[(1-\bar{w}) \frac{\partial \bar{p}}{\partial \bar{t}}-\bar{p} \frac{\partial \bar{w}}{\partial \bar{t}}\right] \\
& \bar{p}(\bar{x}, 0, \bar{t})=\bar{p}(\bar{x}, 1, \bar{t})=\bar{p}(1, \bar{y}, \bar{t})=1, \partial \bar{p}(0, \bar{y}, \bar{t}) / \partial \bar{x}=0
\end{aligned}
$$

where $\hat{P}=l^{4} b p_{a} / E I d_{0}, \beta=l / b, \alpha=\varepsilon_{0} b l^{4} / E I d_{0}{ }^{3}$. The response of the microcantilever $\bar{w}(\bar{x}, \bar{t})$ under an excitation voltage comprising of a large DC voltage and a small AC component can be expressed as

$$
\bar{w}(\bar{x}, \bar{t})=\bar{w}_{0}(\bar{x})+\bar{w}_{d}(\bar{x}, \bar{t})
$$

where $\bar{w}_{0}(\bar{x})$ represents the static deflection under the DC bias voltage $V_{D C}$, and, $\bar{w}_{d}(\bar{x}, \bar{t})$ represents the small amplitude vibration around the deflected position.

\subsection{Static deflection}

Substituting Eq. (6) in Eq. (4) and dropping the dynamic terms, the equation for static response can be obtained as

$$
\frac{d^{4} \bar{w}_{0}}{d \bar{x}^{4}}=\frac{1}{2} \frac{\alpha V_{D C}^{2}}{\left(1-\bar{w}_{0}\right)^{2}}
$$

with the boundary conditions as $\bar{w}_{0}(0)=\left(d \bar{w}_{0} / d \bar{x}\right)_{\bar{x}=0}=\left(d^{2} \bar{w}_{0} / d \bar{x}^{2}\right)_{\bar{x}=1}=\left(d^{3} \bar{w}_{0} / d \bar{x}^{3}\right)_{\bar{x}=1}=0$

Using Galerkin discretization, $\bar{w}_{0}(\bar{x})$ can be approximated as

$$
\bar{w}_{0}(\bar{x})=\sum_{i=1}^{N} a_{i} \phi_{i}(\bar{x})
$$

where $N$ represents the number of modes retained in the solution; $a_{i}$ are the unknown coefficients to be determined. $\phi_{i}(\bar{x})$ is the $i$ th linear undamped mode shape of the undeflected microcantilever obtained from the following linear undamped eigenvalue problem of a straight beam 


$$
\frac{d^{4} \phi_{i}}{d \bar{x}^{4}}=\left(s \omega_{i}\right)^{2} \phi_{i} \text {, and, } \phi_{i}(0)=\left(d \phi_{i} / d \bar{x}\right)_{\bar{x}=0}=\left(d^{2} \phi_{i} / d \bar{x}^{2}\right)_{\bar{x}=1}=\left(d^{3} \phi_{i} / d \bar{x}^{3}\right)_{\bar{x}=1}=0
$$

It is worth mentioning that $\phi_{i}(\bar{x})$ is normalized such that $\int_{0}^{1} \phi_{i}^{2} d \bar{x}=1$. Multiplying Eq. (7a) by $\left(1-\bar{w}_{0}\right)^{2}$, substituting Eqs. (8) and (9) into the resulting equation, multiplying by $\phi_{n}(\bar{x})$, and integrating the outcome from $\bar{x}=0$ to 1 , the set of coupled nonlinear algebraic equations can be derived as

$$
a_{n}\left(s \omega_{n}\right)^{2}-2 \sum_{i=1}^{N} \sum_{j=1}^{N} a_{i} a_{j}\left(s \omega_{i}\right)^{2} \int_{0}^{1} \phi_{n} \phi_{i} \phi_{j} d \bar{x}+\sum_{i=1}^{N} \sum_{j=1}^{N} \sum_{k=1}^{N} a_{i} a_{j} a_{k}\left(s \omega_{i}\right)^{2} \int_{0}^{1} \phi_{n} \phi_{i} \phi_{j} \phi_{k} d \bar{x}-\frac{\alpha V_{D C}}{2} \int_{0}^{1} \phi_{n} d \bar{x}=0, \quad n=1,2, \ldots . ., N
$$

Prediction of the static deflected shape for different bias voltages is very crucial to estimate the characteristics of the resonator in the entire working range of voltage polarization. Reduced order models (ROM) derived in recent works [Chaterjee and Pohit 2009] have shown that at least five modes ( $\mathrm{N}=5$ ) are required in Eqs. (8) and (10) to get the correct deflected shape close to pull-in for beam based MEMS devices even for small air-gap to length ratios. Li et al [2007] used only the first model function $(\mathrm{N}=1)$ and hence the model may be more appropriate for small electrostatic loads.

\subsection{Small amplitude motion}

For small amplitude motion, $\bar{w}_{d}<<\bar{w}_{0}$, the pressure variation in the squeeze-film is also small and the absolute pressure underneath the vibrating plate can be written as

$$
\bar{p}(\bar{x}, \bar{y}, \bar{t})=1+P^{*}(\bar{x}, \bar{y}, \bar{t})
$$

where $\left|P^{*}(\bar{x}, \bar{y}, \bar{t})\right|<<1$. Substituting Eq. (6) and Eq. (11) in Eq. (4a), expanding the excitation term in a Taylor series around $\bar{w}_{0}(\bar{x})$ up to first order, and using the static equilibrium Eq. (7a), the equation for dynamic deflection can be expressed as

$$
\begin{aligned}
& \frac{\partial^{2} \bar{w}_{d}}{\partial \bar{t}^{2}}+\frac{\partial^{4} \bar{w}_{d}}{\partial \bar{x}^{4}}=\frac{\alpha V_{D C}{ }^{2} \bar{w}_{d}}{\left(1-\bar{w}_{0}\right)^{3}}+\frac{\alpha V_{D C} v(\bar{t})}{\left(1-\bar{w}_{0}\right)^{2}}-\hat{P} \int P^{*} d \bar{y} \\
& \bar{w}_{d}(0, \bar{t})=\left(\partial \bar{w}_{d} / \partial \bar{x}\right)_{\bar{x}=0}=0 \text {, and, }\left(\partial^{2} \bar{w}_{d} / \partial \bar{x}^{2}\right)_{\bar{x}=1}=\left(\partial^{3} \bar{w}_{d} / \partial \bar{x}^{3}\right)_{\bar{x}=1}=0
\end{aligned}
$$

Using Eq. (11), Eq. (5) can be linearized as

$$
\begin{aligned}
& \left(1-\bar{w}_{0}\right)^{3} \frac{\partial^{2} P^{*}}{\partial \bar{x}^{2}}-3\left(1-\bar{w}_{0}\right)^{2} \frac{\partial P^{*}}{\partial \bar{x}} \frac{d \bar{w}_{0}}{d \bar{x}}+\beta^{2}\left(1-\bar{w}_{0}\right)^{3} \frac{\partial^{2} P^{*}}{\partial \bar{y}^{2}}-\left(1-\bar{w}_{0}\right) \frac{\Pi}{s} \frac{\partial P^{*}}{\partial \bar{t}}+\frac{\Pi}{s} \frac{\partial \bar{w}_{d}}{\partial \bar{t}} \\
& +\left(1-\bar{w}_{0}\right)^{3} \frac{\partial P^{*}}{\partial \bar{x}} \frac{(9.638)(1.159) K n^{0.159}}{1+9.638 K n^{1.159}} \frac{\partial K n}{\partial \bar{x}}=0 \\
& P^{*}(\bar{x}, 0, \bar{t})=P^{*}(\bar{x}, 1, \bar{t})=P^{*}(1, \bar{y}, \bar{t})=0, \partial P^{*}(0, \bar{y}, \bar{t}) / \partial \bar{x}=0
\end{aligned}
$$

where the linearized Knudsen number $K n(\bar{x})=\lambda_{a} /\left[d_{0}\left(1-\bar{w}_{0}\right)\right] ; \quad \Pi=12 \mu_{\text {eff }} l^{2} / d_{0}{ }^{2} p_{a}$. For a harmonic excitation $v(\bar{t})=v_{0} e^{j(s \omega) \bar{t}}$ the steady-state solution of Eq. (12) and Eq. (13) can be expressed as

$$
\bar{w}_{d}(\bar{x}, \bar{t})=A \phi(\bar{x}) e^{j(s \omega) \bar{t}}, \text { and, } P^{*}(\bar{x}, \bar{y}, \bar{t})=\psi(\bar{x}, \bar{y}) e^{j(s \omega) \bar{t}}
$$

where $A$ represents the complex amplitude of small vibration; $\phi(\bar{x})$ represent the exact mode shapes of the undeflected cantilever beam in flexural modes of vibration. Li et al [2007] represented the spatial pressure distribution $\psi(\bar{x}, \bar{y})$ as a product of an assumed parabolic function along beam width and a cosine series along beam length. Pandey and Pratap [2007] expanded it using Green's function approach of assumed double cosine series. In the present paper, following Li et al [2007], the spatial distribution of pressure $\psi(\bar{x}, \bar{y})$ satisfying the boundary conditions (Eq. 13b) is given as

$$
\psi(\bar{x}, \bar{y})=\sum_{m=1}^{M} z_{m} \cos \left[(2 m-1) \frac{\pi}{2} \bar{x}\right]\left(\bar{y}-\bar{y}^{2}\right)
$$

where $z_{m}$ represent the unknown complex coefficient to be determined, and $M$ represents the number of terms retained in the expansion of the pressure function. Substituting Eqs. (14), (15), and (16) into Eq. (13a) and integrating across the width of the beam leads to

$$
\sum_{m=1}^{M} \frac{z_{m}}{A} U_{m}(\bar{x})=\phi(\bar{x})
$$


where $U_{m}(\bar{x})=u_{m}(\bar{x}) \cos \left[(2 m-1) \frac{\pi}{2} \bar{x}\right]+\bar{u}_{m}(\bar{x}) \sin \left[(2 m-1) \frac{\pi}{2} \bar{x}\right]$

$$
\begin{aligned}
& u_{m}(\bar{x})=\left[(1 / 6)\left\{(2 m-1) \frac{\pi}{2}\right\}^{2}\left(1-\bar{w}_{0}\right)^{3}+2 \beta^{2}\left(1-\bar{w}_{0}\right)^{3}+\frac{1}{6} j \omega \Pi\left(1-\bar{w}_{0}\right)\right] / j \omega \Pi \\
& \bar{u}_{m}(\bar{x})=\frac{-(2 m-1) \frac{\pi}{4}\left(1-\bar{w}_{0}\right)^{2} \frac{d \bar{w}_{0}}{d \bar{x}}+\frac{1}{6}\left\{(2 m-1) \frac{\pi}{2}\right\}\left(1-\bar{w}_{0}\right)^{3} \frac{(9.638)(1.159) K n^{0.159}}{1+9.638 K n^{1.159}} \frac{\partial K n}{\partial \bar{x}}}{j \omega \Pi}
\end{aligned}
$$

Multiplying each term in Eq. (17) by $\cos \left[(2 n-1) \frac{\pi}{2} \bar{x}\right]$ for $n=1,2, \ldots \ldots, M$, and integrating along the beam length leads to the matrix formulation

$$
\frac{1}{A}[D]_{M \times M}[Z]_{M \times 1}=[H]_{M \times 1}
$$

where $\quad D(n, m)=\int_{0}^{1} U_{m}(\bar{x}) \cos \left[(2 n-1) \frac{\pi}{2} \bar{x}\right] d \bar{x}$

$$
\begin{aligned}
& H(n)=\int_{0}^{1} \phi(\bar{x}) \cos \left[(2 n-1) \frac{\pi}{2} \bar{x}\right] d \bar{x} \\
& Z(m)=z_{m}{ }^{R}+j z_{m}{ }^{I}
\end{aligned}
$$

where $z_{m}{ }^{R}$ and $z_{m}{ }^{I}$ are the real and imaginary parts, respectively. Using Eqs. (15) and (16), the distributed force acting on the beam owing to the time varying pressure distribution underneath it can be rewritten as

$$
\hat{P}_{0}^{1} P^{*} d \bar{y}=A e^{j(s \omega) \bar{t}}\left[F_{S}(\bar{x})+j F_{d}(\bar{x})\right]
$$

where $\quad F_{s}(\bar{x})=\frac{\hat{P}}{6} \sum_{m=1}^{M} \frac{z_{m}{ }^{R}}{A} \cos \left[(2 m-1) \frac{\pi}{2} \bar{x}\right]$, and, $\quad F_{d}(\bar{x})=\frac{\hat{P}}{6} \sum_{m=1}^{M} \frac{z_{m}{ }^{I}}{A} \cos \left[(2 m-1) \frac{\pi}{2} \bar{x}\right]$

The spring effect of the trapped air is represented by $F_{s}(\bar{x})$ while $F_{d}(\bar{x})$ stands for the damping effect. The relative dominance of the elastic force and the viscous damping force due to the squeeze-film can be obtained by calculating the ratio $\Gamma$ (hereafter called the compressibility ratio) given by

$$
\Gamma=\int_{0}^{1} F_{s}(\bar{x}) d \bar{x} / \int_{0}^{1} F_{d}(\bar{x}) d \bar{x}
$$

For compressibility ratio $\Gamma<<1$, the elastic forces are negligible and the flow can be assumed to be incompressible whereas for finite values of $\Gamma$ compressibility effects cannot be ignored. For $\Gamma>1$, spring effects dominate over the damping effects of the squeeze-film.

Substituting Eqs. (14), (15), and (25) into Eq. (12a), multiplying throughout by $\phi(\bar{x})$, and integrating from $\bar{x}=0$ to 1 while using the normalization condition, complex amplitude $A$ can be obtained as

$$
A=\frac{\alpha V_{D C} v_{0} \int_{0}^{1} \frac{\phi(\bar{x})}{\left(1-\bar{w}_{0}(\bar{x})\right)^{2}} d \bar{x}}{\left\{\left(s \omega_{i}\right)^{2}-k_{e}+k_{a}\right\}\left[1-\frac{\omega^{2}}{\omega_{i}^{2}} \frac{\left(s \omega_{i}\right)^{2}}{\left(s \omega_{i}\right)^{2}-k_{e}+k_{a}}+j \frac{\omega}{\omega_{i}} \frac{c}{\left(s \omega_{i}\right)^{2}-k_{e}+k_{a}}\right]}
$$

where $\quad k_{e}=\alpha V_{D C} \int_{0}^{1} \frac{\phi^{2}(\bar{x})}{\left(1-\bar{w}_{0}(\bar{x})\right)^{3}} d \bar{x}$

represents the spring softening effect due to electrostatic coupling. The squeeze-film stiffness $k_{a}$ and the damping coefficient $c$ are given by

$$
k_{a}=\int_{0}^{1} F_{s}(\bar{x}) \phi(\bar{x}) d \bar{x}=\frac{\hat{P}}{6} \sum_{m=1}^{M} \frac{z_{m}{ }^{R}}{A} H(m)
$$




$$
c=\frac{\omega_{i}}{\omega} \int_{0}^{1} F_{d}(\bar{x}) \phi(\bar{x}) d \bar{x}=\frac{\omega_{i}}{\omega} \frac{\hat{P}}{6} \sum_{m=1}^{M} \frac{z_{m}{ }^{I}}{A} H(m)
$$

The overall stiffness of the system is governed by the relative magnitudes of $k_{e}$ and $k_{a}$. Using Eq. (29), the quality factor $Q$ and the resonance frequency $\omega_{\text {res }}$ of the microcantilever resonator under squeeze-film damping can be expressed as

$$
Q=1 / 2 \xi=\left(s \omega_{i}\right)\left(\sqrt{\left(s \omega_{i}\right)^{2}-k_{e}+k_{a}}\right) / c \text {, and, } \omega_{\text {res }}=\frac{1}{s} \sqrt{\left(s \omega_{i}\right)^{2}-k_{e}+k_{a}-\frac{c^{2}}{2\left(s \omega_{i}\right)^{2}}}
$$

where $\xi$ is the damping ratio.

\subsection{Incompressible flow model}

The present section assumes an incompressible and non-inertial flow in the air-gap. As the flow is incompressible, the squeezefilm spring forces will be absent and there will be only dissipation of the movement. Proceeding as in section 2.1 , the nonlinear incompressible Reynolds equation can be expressed in the nondimensional form as

$$
\begin{aligned}
& \frac{\partial}{\partial \bar{x}}\left[\frac{1}{\mu_{e f f}}(1-\bar{w})^{3} \frac{\partial \bar{p}}{\partial \bar{x}}\right]+\beta^{2} \frac{\partial}{\partial \bar{y}}\left[\frac{1}{\mu_{e f f}}(1-\bar{w})^{3} \frac{\partial \bar{p}}{\partial \bar{y}}\right]=\frac{12 l^{2}}{d_{0}{ }^{2} p_{a} s}\left[-\frac{\partial \bar{w}}{\partial \bar{t}}\right] \\
& \bar{p}(\bar{x}, 0, \bar{t})=\bar{p}(\bar{x}, 1, \bar{t})=\bar{p}(1, \bar{y}, \bar{t})=1, \partial \bar{p}(0, \bar{y}, \bar{t}) / \partial \bar{x}=0
\end{aligned}
$$

As explained in section 2.3, Eq. (35a) can be linearized and expressed as Eq. (13a) with the exclusion of the term $\left(1-\bar{w}_{0}\right) \frac{\Pi}{s} \frac{\partial P^{*}}{\partial \bar{t}}$. For incompressible flow, Eq. (19) can be modified by excluding the term $\frac{1}{6} j \omega \Pi\left(1-\bar{w}_{0}\right)$. Repeating the rest of the procedure as described in section 2.3, the quality factor $Q$ and the resonance frequency $\omega_{\text {res }}$ can be expressed by Eqs. (33) and (34) respectively.

\section{Finite Element Model}

The squeeze-film damping has been evaluated using the modal projection technique available in the FEA software package ANSYS. Three coupled-field domain solvers: electrostatic, structural, and fluid have been utilized. The structural domain has been modeled with SOLID 45 elements used for 3-D modeling of solid structures, and, capable of modeling stress stiffening associated with static deflection due to large DC bias voltages. Direct coupling of electrostatic and structural domain has been achieved using TRANS 126 elements. TRANS 126 elements are lumped electromechanical transducers preferable for highly non-linear coupledfield interaction. The model uses FLUID 136 elements to account for the squeeze-film damping effect which is based on linearized compressible Reynolds equation modified for the rarefied gas effect and is thus applicable for small pressure changes in the airgap. At first, a pre-stress static analysis with an applied DC voltage is carried out to obtain the static bias deflection. The local gap separation treated as a real constant for each fluid element is updated to reflect the resultant non-uniform gap spacing. An effective viscosity $\mu_{\text {eff }}$ being dependent on the variable Knudsen number $K n$ is assigned to each fluid element with the updated local gap separation. A modal analysis is therefore performed to compute the natural frequencies and the mode shapes (eigenvectors) of the flexural modes of vibration. A squeeze-film analysis can be performed on structures with known velocities or unknown velocities. For a beam undergoing flexible body dynamics, the displacement and velocity vary along the structure. Thus, the fluid film underneath the beam is subjected to varying velocity profile. In case of modal projection method, the harmonic analysis on the thin-film fluid elements is performed with the nodal velocities equal to the eigenvector of the appropriate mode. The resulting pressure distribution and hence the modal forces are computed to obtain the modal squeeze stiffness and damping parameters. Subsequently, the modal damping ratio is obtained corresponding to the flexural modes.

\section{Results and Discussion}

The entire analysis is carried out following the two procedures outlined in the previous sections, namely, semi-analytical method and finite element method. The semi-analytical method uses the compressible flow model to generate the results presented in this section. A comparison of the squeeze-film characteristics obtained with the incompressible and compressible flow models has also been presented in section 4.3. In this paper, $M$ is taken as 25 which exhibit good convergence even at large DC voltages. First, the converged results are validated with the analytical, numerical and experimental results available in open literature. The design properties used in the present analysis are $l=350 \mu \mathrm{m}, b=22 \mu \mathrm{m}, h=4 \mu \mathrm{m}, d_{0}=1.4 \mu \mathrm{m}, \rho=2330 \mathrm{~kg} / \mathrm{m}^{3}, E=160 \mathrm{e} 9 \mathrm{~Pa}$, $p_{a}=1.013 e 5 \mathrm{~Pa}, \mu=1.8 e-5 \mathrm{Ns} / \mathrm{m}^{2}$ as used for the experimental study by Pandey and Pratap [2007]. The effects of large DC bias 
voltages on resonance frequency and quality factor are studied next for the first three flexural modes of vibration of the resonator. Variations of ambient pressure in presence of DC bias voltages are shown to affect the resonance frequency and the quality factor of the microresonator.

The unknown coefficients $a_{i}$ present in Eq. (8) are evaluated by numerically solving the set of nonlinear algebraic equations obtained retaining five ( $\mathrm{N}=5$ ) modes in Eq. (10). The static deflection for a DC bias voltage is then expressed as a function of position $\bar{x}$ using Eq. (8). The spring softening effect $k_{e}$, squeeze-film stiffness $k_{a}$, and the damping coefficient $c$ are obtained using Eqs. (21)-(23) and (30)-(32) where the integrations are numerically obtained. Thereafter, using Eqs. (33) and (34), the quality factor $Q$, the damping ratio $\xi$, and the resonance frequency $\omega_{\text {res }}$ are calculated for the flexural modes of vibration. The undamped natural frequency is calculated from Eq. (34) replacing terms $k_{a}$ and $c$ by zero.

To validate the present approaches, the damping ratio $\xi$ results obtained with the present methods for zero $\left(V_{D C}=0\right)$ DC voltage are compared with the results obtained by Pandey and Pratap [2007]. As shown in Table 1, the results for the cantilever microresonator are in excellent agreement for all the three modes of vibration.

Table 1. Comparison of the damping ratio $\xi$ results for zero $\left(V_{D C}=0\right)$ DC bias voltage.

\begin{tabular}{ccccccc}
\hline $\begin{array}{c}\text { Length } \\
l \\
(\mu \mathrm{m})\end{array}$ & $\begin{array}{c}\text { Flexural } \\
\text { mode }\end{array}$ & $\begin{array}{c}\text { Experimental } \\
\text { [Pandey and } \\
\text { Pratap 2007] }\end{array}$ & $\begin{array}{c}\text { FE model } \\
\text { [Pandey and } \\
\text { Pratap 2007] }\end{array}$ & $\begin{array}{c}\text { FE model } \\
\text { [Present } \\
\text { Work] }\end{array}$ & $\begin{array}{c}\text { Analytical } \\
\text { [Pandey and } \\
\text { Pratap 2007] }\end{array}$ & $\begin{array}{c}\text { Present } \\
\text { model }\end{array}$ \\
\hline \multirow{3}{*}{350} & $1^{\text {st }}$ & $0.415 \pm 0.002$ & 0.45 & 0.4475 & 0.422 & 0.4484 \\
& $2^{\text {nd }}$ & $0.066 \pm 0.002$ & 0.072 & 0.0710 & 0.067 & 0.0713 \\
& $3^{\text {rd }}$ & $0.027 \pm 0.002$ & 0.025 & 0.0244 & 0.024 & 0.0247 \\
\hline
\end{tabular}

\subsection{Small amplitude motion under DC bias voltage}

Next, the effect of finite DC bias voltages is studied using both the semi-analytical model and the FE model, and, the results are compared.

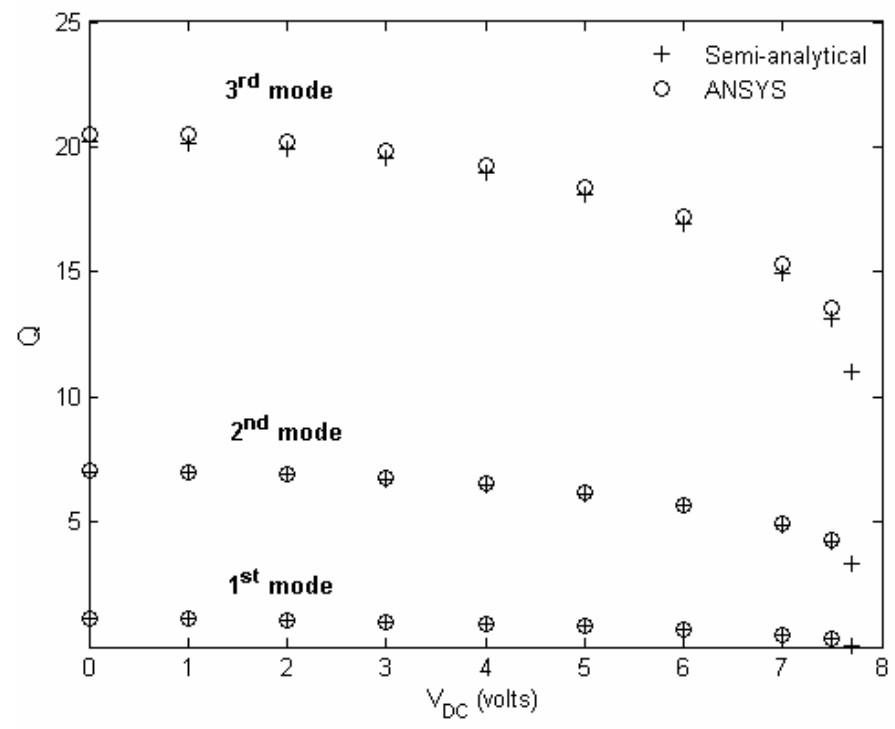

Figure 2. Variations of quality factor $Q$ with DC voltage $V_{D C}$ for the first three flexural modes.

At a certain DC bias voltage, the quality factor $Q$ is higher for higher mode of vibration (Fig. 2). Even at large DC voltages (except at pull-in) results obtained with the semi-analytical model compares very well with the ANSYS results. As shown in Fig. 2, for a certain mode of vibration, the quality factor decreases monotonically with DC voltages. However, the percentage decrease in quality factor $\Delta Q\left(\left|\left(Q-Q_{\text {ref }}\right)\right| \times 100 / Q_{\text {ref }}\right)$ for a certain increase in DC voltage $\Delta V\left(V_{D C}-V_{\text {ref }}\right)$ is observed (Fig. 3) to be higher for the first mode of vibration in comparison to the higher modes. As shown in Fig. 3, the quality factor decreases sharply $(\approx 75 \%)$ for the first mode as pull-in is approached. 


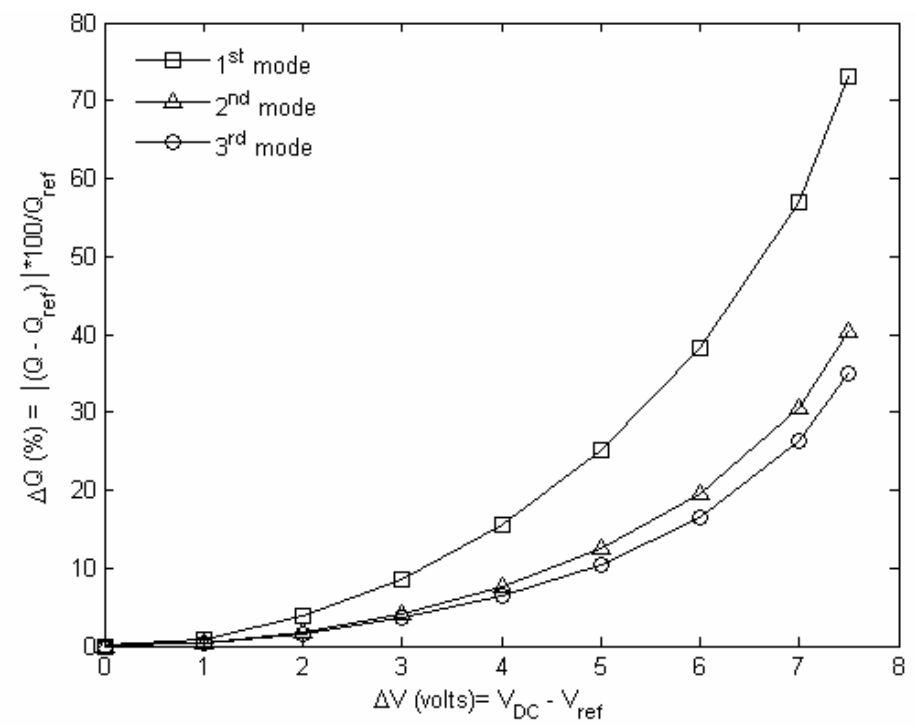

Figure 3. Effect of variations in DC bias voltage $\Delta V$ on percentage decrease in quality factor $\Delta Q$ for the first three flexural modes. The reference ( ref ) values correspond to $V_{D C}$ equal to zero.

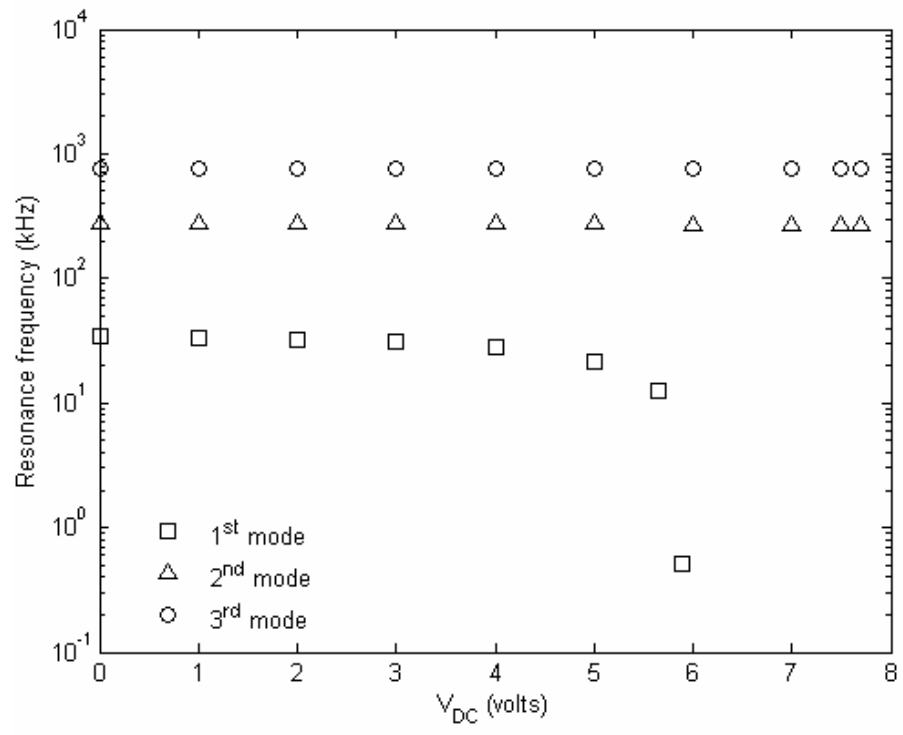

Figure 4. Variations of resonance frequency with DC voltage $V_{D C}$ for the first three flexural modes.

Figure 4 shows the variations of the resonance frequency for the first three flexural modes of vibration of the resonator. For the first mode of vibration of the resonator operating under the present ambient $\left(p_{a}=1.013 e 5 P a\right)$ conditions, the resonant peak disappears at bias voltages ( $V_{D C} \approx 6$ volts) much lower than the static pull-in ( $V_{D C}=7.704$ volts) voltage due to increase in damping thus limiting the working range of voltage polarizations for the resonator. Though the resonance frequency for the first mode decreases monotonically with DC bias, the resonance frequency for the higher modes is more or less uniform for the entire range of DC polarization. With increase in static bias voltage the gap separation decreases resulting in an increase in the damping effect $c$ as well as stiffening effects $k_{a}$ of the squeeze-film. Further, the electrostatic softening effects $k_{e}$ are pronounced at large DC voltages. However, the magnitude of these variations with DC bias voltage differs considerably for different modes of vibration which ultimately shows up in the computed quality factors and the resonance frequencies of the resonator. For the beam properties considered, the variations in $k_{e}, k_{a}$ and $c$ with DC voltage are such that the resonance frequency for the analyzed higher modes is nearly unaffected in the entire range of DC polarization. Further, as shown in Fig. 5, the DC bias voltage in conjunction with the mode shape has a profound effect on the pressure distribution in the fluid film underneath the beam. Figure 5 obtained with ANSYS clearly show the difference in the dynamic pressure (relative) variation in the first three flexural modes of vibration for two different bias voltages $V_{D C}=0$ volts and $V_{D C}=7.5$ volts. For each mode, the peak back pressure on the bottom 
surface of the moving structure is higher at larger bias voltage, and, the high pressure zone is more localized near the tip of the cantilever beam. This difference in pressure distribution affects the squeeze-film characteristics of the flexible beam.

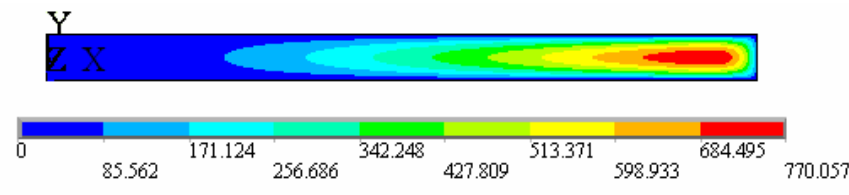

(a)

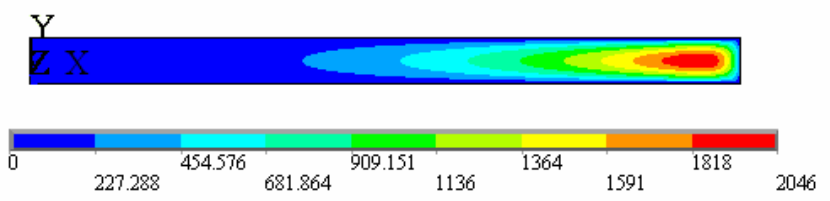

(b)

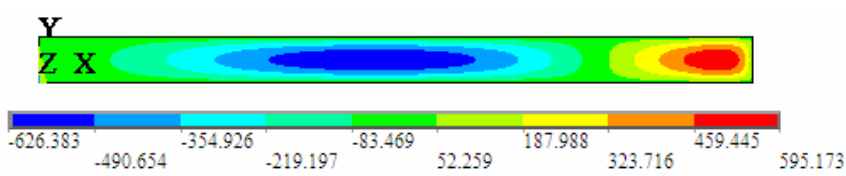

(a)

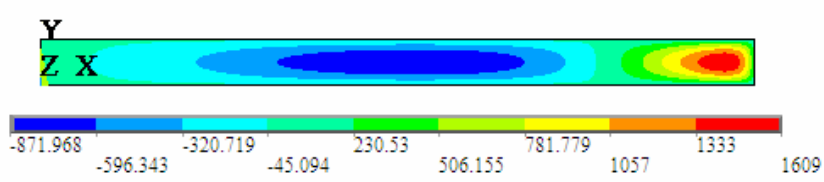

(b)

(II) Second mode

(I) First mode

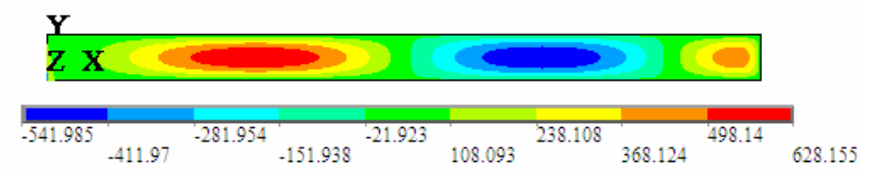

(a)

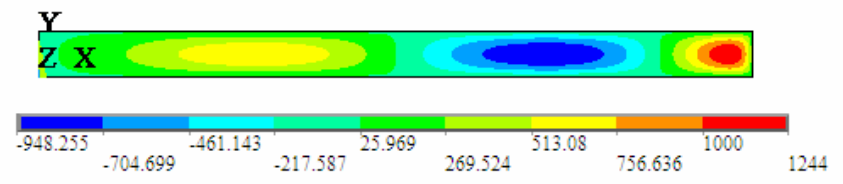

(b)

(III) Third mode

Figure 5. Pressure distribution (in Pa) on the bottom surface of the resonator actuated by (a) $V_{D C}=0$ volts and (b) $V_{D C}=7.5$ volts.

\subsection{Effects of variations in ambient pressure}

The effects of DC voltage with varying gap pressure on quality factor and resonance frequency are investigated next. Pressure dependency of the damping has been analyzed with the model by considering the pressure dependency of the mean free path $\lambda_{a}$.

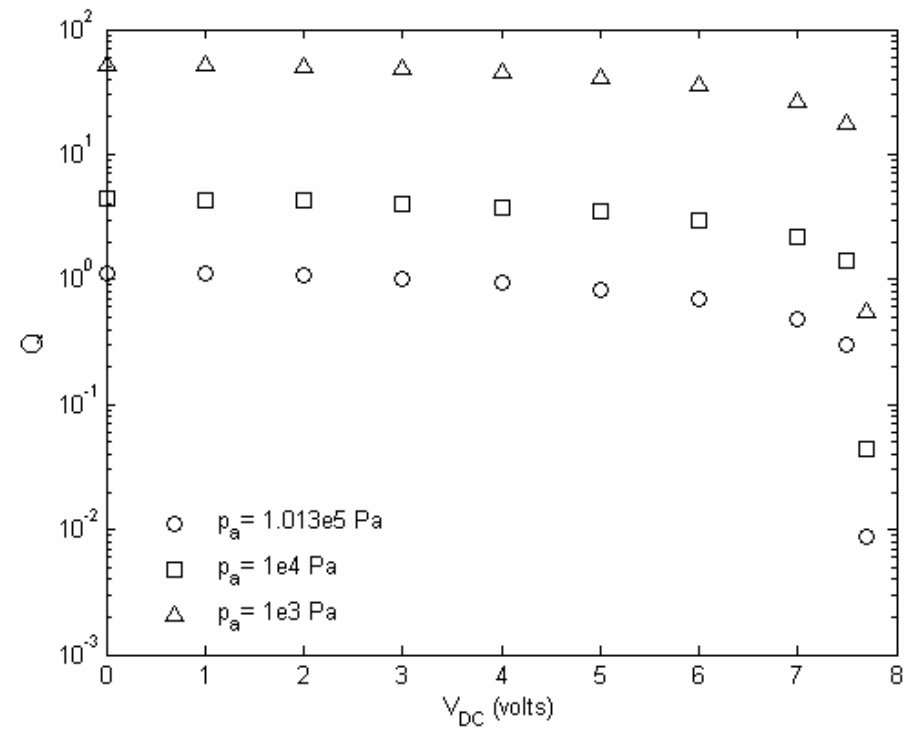

(I) First mode

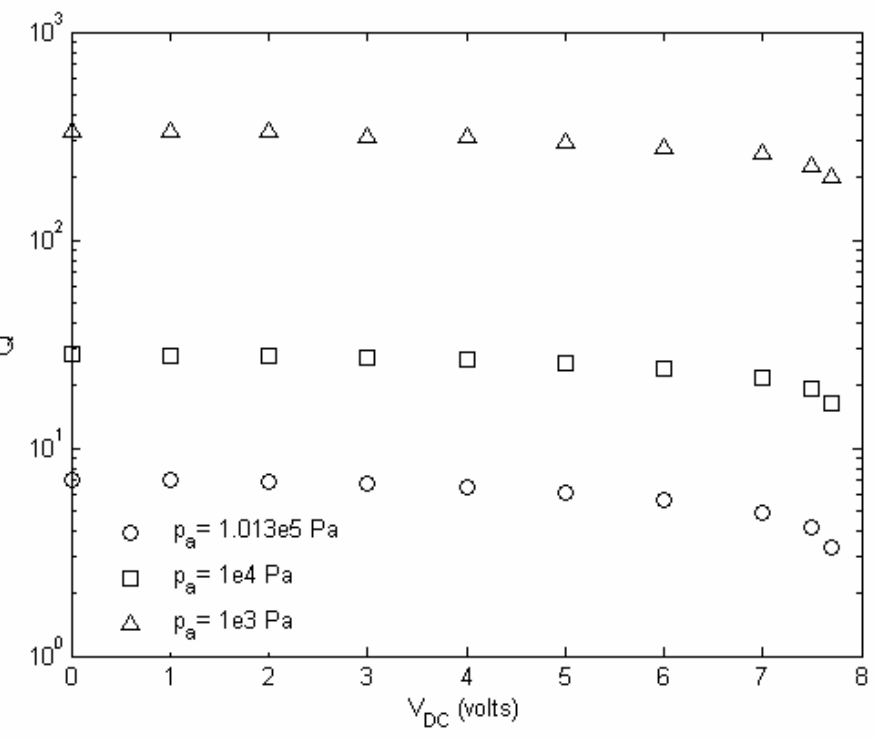

(II) Second mode 


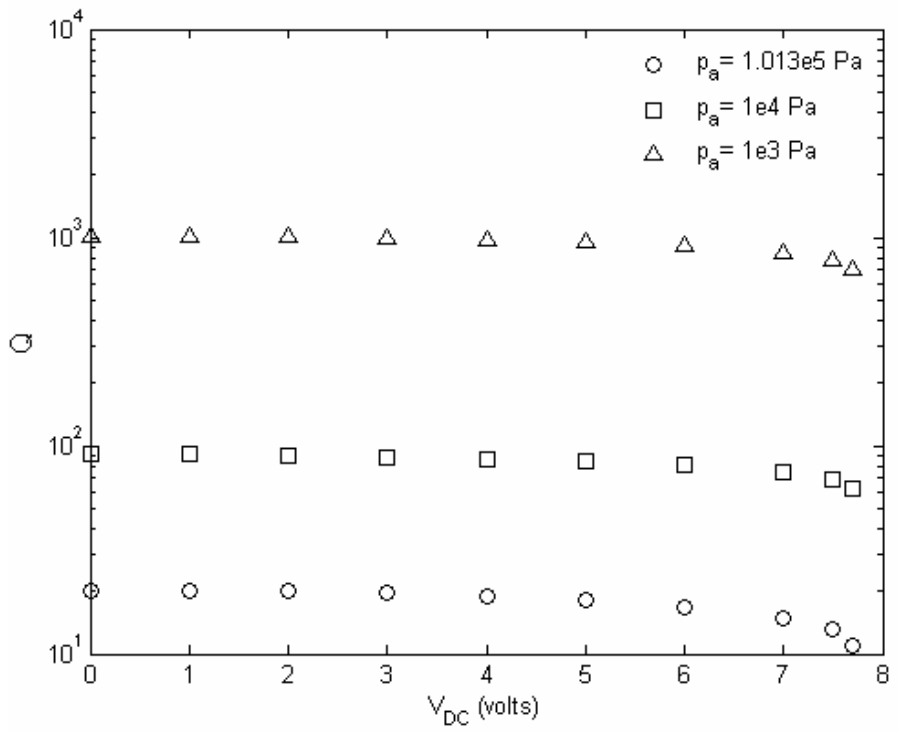

(III) Third mode

Figure 6. Variations of quality factor $Q$ with DC voltage $V_{D C}$ for various ambient air pressures.

Three ambient pressure conditions $p_{a}=1.013 e 5 \mathrm{~Pa}, p_{a}=1 e 4 \mathrm{~Pa}$, and, $p_{a}=1 e 3 \mathrm{~Pa}$ have been considered in this section. As shown in Fig. 6, quality factor for all the three modes increases with decrease in gap pressure as the effect of squeeze-film damping decreases. It is also observed that at a certain DC voltage $V_{D C}$, the increase in quality factor with decrease in pressure is more or less uniform for the entire range of DC polarization.

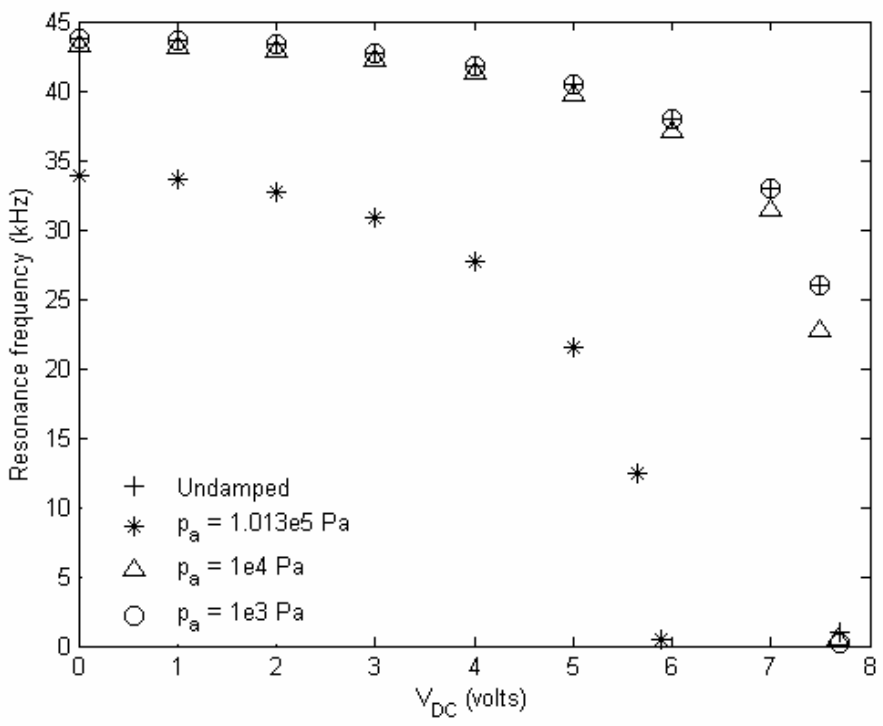

Figure 7. Variations of resonance frequency with DC voltage $V_{D C}$ for the first mode for various ambient air pressures.

Figure 7 shows variation of the resonance frequency with DC voltage $V_{D C}$ for the first mode of vibration of the resonator for various values of ambient pressure $p_{a}$. As shown in Fig. 7, at a certain DC voltage the undamped and damped frequencies have no appreciable difference at low pressures. Thus, it can be concluded that the squeeze-film damping at low pressures has negligible effect on first mode resonance frequency for the entire range of DC polarization. For the higher modes of vibration, resonance frequency is observed (not shown here) to be nearly invariant with pressure variations for the entire range of DC polarization.

\subsection{Comparison between incompressible and compressible flow models}

To illustrate the effect of compressibility, a comparison of the quality factors computed using the present semi-analytical method based on compressible and incompressible flow has been presented in this section. 


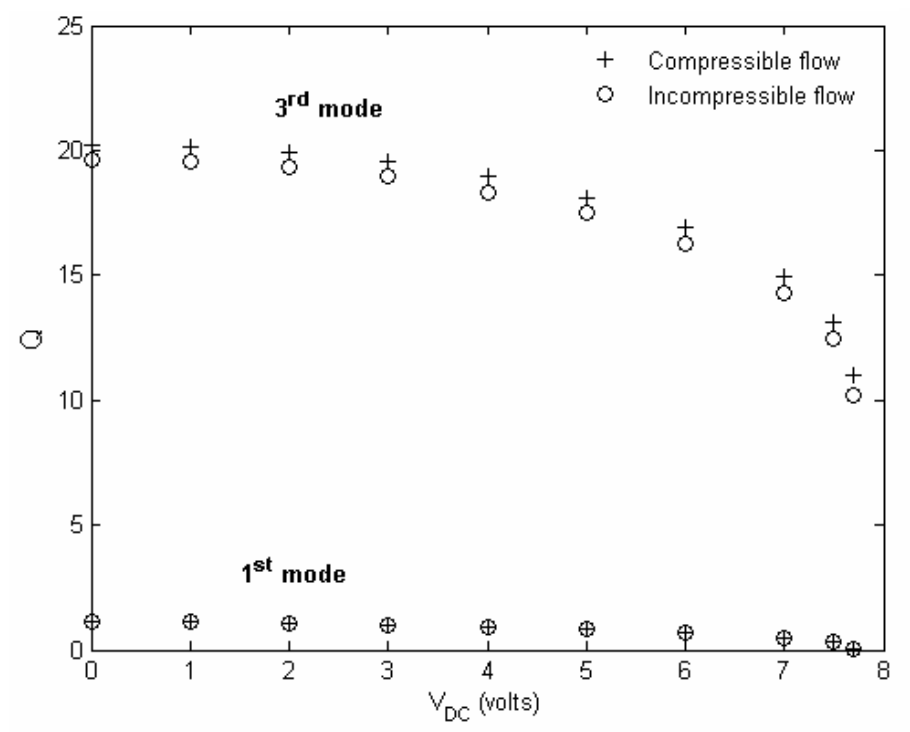

(a) $p_{a}=1.013 e 5 \mathrm{~Pa}$

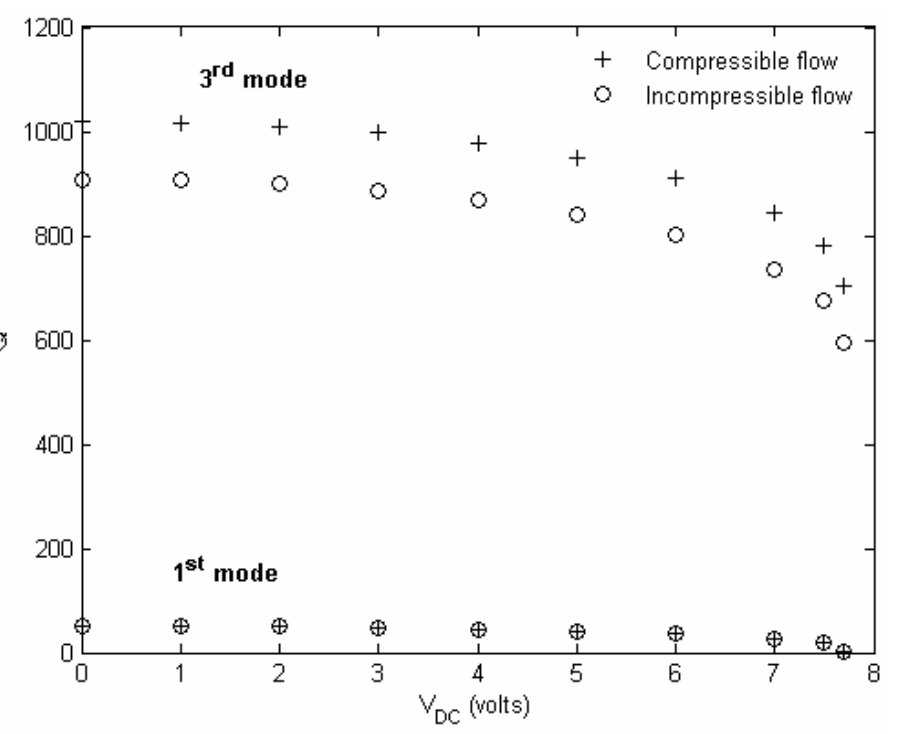

(b) $p_{a}=1 e 3 P a$

Figure 8. Variations of quality factor $Q$ with DC voltage $V_{D C}$ for the different flow models for different ambient pressures.

As shown in Fig. 8(a), no appreciable difference exists in the quality factor values obtained with the compressible and incompressible flow models. The compressibility ratio $\Gamma$ values $(\approx 0.009$ for the first mode and, $\approx 0.02$ for the third mode) computed for case (a) explains the negligible compressible effects. However, Fig. 8(b) shows appreciable difference in the quality factor values obtained with the two flow models for the third mode $(\Gamma \approx 0.34)$ of vibration. Thus, it can be concluded that the difference between the incompressible and compressible flow models used in predicting the squeeze-film characteristics becomes more pronounced as the compressibility ratio $\Gamma$ increases. Moreover, as shown in Fig. 8(b), quality factors obtained with the incompressible flow model is comparatively lower for the entire range of DC polarization as squeeze-film stiffening effects are absent for incompressible flow and the damping is overestimated. For brevity, only the first and third mode results have been presented in this section.

\section{Conclusions}

A semi-analytical model taking into account the static deflection due to large DC bias voltages has been used to study the squeeze film damping characteristics of cantilever microresonator for the first three flexural modes of vibration in the rarefied and non-inertial flow regime. Elastic beam equation for small air-gap coupled with linearized compressible Reynolds equation has been used to model the small amplitude motions. The pressure function assumed as a product of a parabolic function along the beam width and a cosine series along the beam length gives converged results for computationally reasonable number of pressure terms. Dependency of the effective viscosity on the gap height has been considered through the use of a variable Knudsen number. The model is validated by comparing the results with the analytical, numerical, and experimental results available in open literature as well as through ANSYS simulations. The results show that the quality factor is higher for higher mode of vibration for the entire range of DC polarization. With variations in DC bias voltage, quality factor and resonance frequency are most affected in the first mode and are found to decrease with an increase in DC voltage. For flexible microbeams, DC bias voltage in conjunction with mode shape has a profound effect on the back pressure distribution which ultimately affects the squeeze-film characteristics.

The present work also focuses on a range of ambient pressures limited to the viscous flow regime considering rarefaction effects. Under rarefied flow conditions, the results show that at a certain DC voltage the quality factor increases with decrease in ambient pressure and the amount of increase is more or less uniform for the entire range of DC polarization. On the other hand, for low ambient pressures, there is no sufficient change in resonance frequency to be useful as a response characteristic of the resonator (e.g., pressure sensor) at the first harmonic. For higher modes, the resonance frequency is found to be invariant with pressure as well as DC voltage variations. Further, a comparison of quality factor values computed with compressible and incompressible flow models shows that the incompressible flow model overestimates the damping and the effect is more pronounced for higher compressibility ratio. 


\section{Nomenclature}

$\begin{array}{llll}l & \text { Length of the beam } & \mu & \text { Coefficient of viscosity } \\ b & \text { Width of the beam } & \mu_{e f f} & \text { Effective coefficient of viscosity } \\ h & \text { Beam thickness } & \lambda_{a} & \text { Mean free path at ambient pressure } \\ d_{0} & \text { Air-gap } & K n & \text { Knudsen number } \\ X, y, z & \text { Length, width and thickness co-ordinates } & p & \text { Absolute pressure in the air-gap } \\ I & \text { Area moment of inertia } & p_{a} & \text { Ambient pressure } \\ A & \text { Cross sectional area } & \hat{P} & \text { Non-dimensional pressure } \\ \beta & \text { Aspect ratio } & P^{*} & \text { Small pressure variation } \\ w & \text { Beam deflection } & \psi & \text { Pressure function } \\ w_{0} & \text { Static deflection } & z_{m} & \text { Unknown coefficients in the pressure } \\ w_{d} & \text { Small dynamic deflection } & & \text { function } \\ g & \text { Non-uniform gap spacing } & z_{m}{ }^{R}, z_{m}{ }^{I} & \text { Real and imaginary parts of } z_{m} \\ t & \text { Time } & f_{d} & \text { Damping force } \\ V(t) & \text { Excitation voltage } & F_{s} & \text { Spring force due to the squeeze-film } \\ V_{D C} & \text { DC voltage } & F_{d} & \text { Damping force due to the squeeze-film } \\ v & \text { Small AC voltage } & M & \text { Number of terms retained in } \psi \\ v_{0} & \text { Amplitude of AC voltage } & \Gamma & \text { Compressibility ratio } \\ \omega & \text { Excitation frequency } & A & \text { Amplitude of small dynamic deflection } \\ \alpha & \text { Strength of electric actuation } & k_{e} & \text { Electrostatic softening effect } \\ \varepsilon_{0} & \text { Permittivity constant for free space } & k_{a} & \text { Squeeze-film stiffening effect } \\ E^{\prime} & \text { Young's modulus } & c & \text { Squeeze-film damping effect } \\ E & \text { Plate modulus } & \xi & \text { Damping ratio } \\ \rho & \text { Density of beam material } & Q & \text { Quality factor } \\ v & \text { Poisson's ratio } & \omega_{r e s} & \text { Resonance frequency } \\ a_{i} & \text { Unknown coefficients in the static } & & \\ \phi_{i} & \text { deflection function } & \text { Mode shape } & \end{array}$

\section{References}

ANSYS 10, Finite Element Solver for Multiphysics Problems: < http://www.ansys.com/ > .

Batra R.C., Porfiri M. and Spinello D., 2008, Vibrations of narrow microbeams predeformed by an electric field, Journal of Sound and Vibration, vol.309, pp.600-612.

Blech J. J., 1983, On isothermal squeeze films, Journal of Lubrication Technology A, vol.105, pp.615-620.

Chaterjee S. and Pohit G., 2009, A large deflection model for the pull-in analysis of electrostatically actuated microcantilever beams, Journal of Sound and Vibration, vol.322, pp.969-986.

Darling R. B., Hivick C. and Xu J., 1998, Compact analytical modeling of squeeze film damping with arbitrary venting conditions using a Green's function approach, Sensors Actuators A, vol.70, pp.32-41.

Hamrock B.J., 1994, Fundamentals of Fluid Film Lubrication, McGraw-Hill, NY.

Krylov S. and Maimon R., 2004, Pull-in dynamics of an elastic beam actuated by continuously distributed electrostatic force, Journal of Vibration and Acoustics, vol.126, pp.332-342.

Langlois W. E., 1962, Isothermal squeeze films, Quarterly of Applied Mathematics, vol.XX-2, pp. 131- 150.

Li P., Hu R. and Yuming F., 2007, A new model for squeeze-film damping of electrically actuated microbeams under the effect of a static deflection, Journal of Micromechanics and Microengineering, vol.17, pp.1242-1251.

Maali A., Hurth C., Boisgard R., Jai C. and Bouhacina Jean-Pierre Aimé T. C., 2005, Hydrodynamics of oscillating atomic force microscopy cantilevers in viscous fluids, Journal of Applied Physics, vol.97, pp. 074907.

McCarthy B., Adams G., McGruer N. and Potter D., 2002, A dynamic model, including contact bounce, of an electrostatically actuated microswitch, Journal of Microelectromechanical Systems, vol.11, pp.276-283.

Nathanson H.C., Newell W.E., Wickstrom R.A. and Davis J.R., 1967, The resonant gate transistor, IEEE Transactions of Electron Devices, vol.ED-14, No.3, pp.117-133.

Newell W. E., 1968, Miniaturization of tuning forks, Science, vol.161, pp. 1320-1326. 
Nguyen C.T.-C., 2004, Vibrating RF MEMS for next generation wireless applications, Proceedings of the Custom Integrated Circuits Conference, Orlando, FL, 3-6 October. IEEE, Piscataway.

Oden P. I., Chen G. Y., Steele R. A., Warmack R. J. and Thundat T., 1996, Viscous drag measurements utilizing microfabricated cantilevers, Applied Physics Letters , vol.68, pp. 1465-1469.

Paci D., Mastrangeli M., Nannini A. and Pieri F., 2006, Modeling and characterization of three kinds of MEMS resonators fabricated with a thick polysilicon technology, Analog Integr Circ Sig Process, vol.48, pp. 41-47.

Pandey A. K. and Pratap R., 2007, Effect of flexural modes on squeeze film damping in MEMS cantilever resonators, Journal of Micromechanics and Microengineering, vol.17, pp.2475-2484.

Thundat T., Wachter E. A., Sharp S. L. and Warmack R. J., 1995, Detection of mercury vapor using resonating cantilevers, Applied Physics Letters, vol.66, pp. 1695-1697.

Veijola T., Kuisma H., Lahdenper“a J. and Ryh"anen T., 1995, Equivalent-circuit model of the squeezed gas film in a silicon accelerometer, Sensors Actuators A, vol.48, pp.239-248.

Younis M. I. and Nayfeh A. H., 2007, Simulation of squeeze-film damping of microplates actuated by large electrostatic load, ASME Journal of Computational and Nonlinear Dynamics, vol.2, pp.101-112.

Zhang C., Xu G. and Jiang Q., 2004, Characterization of the squeeze film damping effect on the quality factor of a microbeam resonator, Journal of Micromechanics and Microengineering, vol.14, pp.1302-1306.

\section{Biographical notes}

Goutam Pohit is a Professor in Mechanical Engineering, Jadavpur University, Kolkata, India. He did his M.S. (Mechanical Engineering) from the University of Akron, USA, and obtained his Ph.D. from Indian Institute of Technology, Kanpur, India. He has twenty years of teaching experience. His research interest includes non-linear vibration of rotating structures, MEMS and virtual manufacturing. He has written a textbook on CAD published by Pearson Education (Singapore) Pte. Ltd., Indian Branch, Delhi, India. He also has seven years of industrial experience in various capacities.

Saikat Chaterjee received his B.E in Mechanical Engineering from Indira Gandhi Institute of Technology, Sarang, in 1997 and ME in Mechanical Engineering (Machine Design) from Bengal Engineering College (presently BESU), Kolkata, in 2003. He is a faculty member of C. V. Raman College of Engineering, Bhubaneswar and is currently pursuing his Ph.D. in the Department of Mechanical Engineering, Jadavpur University, Kolkata. He is engaged in academic activities since the last 11 years. His research interests include computational analysis of MEMS devices and nonlinear vibration.

Received January 2010

Accepted March 2010

Final acceptance in revised form May 2010 